\title{
Simulations of Intracellular Calcium Release Dynamics in Response to a High-Intensity, Ultrashort Electric Pulse
}

\author{
R. P. Joshi \\ Old Dominion University
}

A. Nguyen
Old Dominion University

V. Sridhara

Old Dominion University

Q. $\mathrm{Hu}$

Old Dominion University

R. Nuccitelli

Old Dominion University

See next page for additional authors

Follow this and additional works at: https://digitalcommons.odu.edu/bioelectrics_pubs

Part of the Biomedical Engineering and Bioengineering Commons

\section{Repository Citation}

Joshi, R. P.; Nguyen, A.; Sridhara, V.; Hu, Q.; Nuccitelli, R.; Beebe, Stephen J.; Kolb, J.; and Schoenbach, Karl H., "Simulations of Intracellular Calcium Release Dynamics in Response to a High-Intensity, Ultrashort Electric Pulse" (2007). Bioelectrics Publications. 42.

https://digitalcommons.odu.edu/bioelectrics_pubs/42

\section{Original Publication Citation}

Joshi, R.P., Nguyen, A., Sridhara, V., Hu, Q. Nuccitelli, R., Beebe, S.J., .. Schoenbach, K.H. (2007). Simulations of intracellular calcium release dynamics in response to a high-intensity, ultrashort electric pulse. Phys Rev E Stat Nonlin Soft Matter Phys, 75(4 Pt 1), 041920. doi:10.1103/PhysRevE.75.041920 
Authors

R. P. Joshi, A. Nguyen, V. Sridhara, Q. Hu, R. Nuccitelli, Stephen J. Beebe, J. Kolb, and Karl H. Schoenbach 


\title{
Simulations of intracellular calcium release dynamics in response to a high-intensity, ultrashort electric pulse
}

\author{
R. P. Joshi, ${ }^{1,2}$ A. Nguyen, ${ }^{1}$ V. Sridhara, ${ }^{1}$ Q. Hu, ${ }^{1}$ R. Nuccitelli, ${ }^{1,2}$ S. J. Beebe, ${ }^{2,3}$ J. Kolb, ${ }^{1,2}$ and K. H. Schoenbach ${ }^{1,2}$ \\ ${ }^{1}$ Department of Electrical and Computer Engineering, Old Dominion University, Norfolk, Virginia 23529-0246, USA \\ ${ }^{2}$ Center for Bioelectrics, Old Dominion University, Norfolk, Virginia 23529-0246, USA \\ ${ }^{3}$ Department of Physiological Science, Eastern Virginia Medical School, Norfolk, Virginia 23501, USA
}

(Received 8 January 2007; revised manuscript received 6 March 2007; published 30 April 2007)

\begin{abstract}
Numerical simulations for electrically induced, intracellular calcium release from the endoplasmic reticulum are reported. A two-step model is used for self-consistency. Distributed electrical circuit representation coupled with the Smoluchowski equation yields the ER membrane nanoporation for calcium outflow based on a numerical simulation. This is combined with the continuum Li-Rinzel model and drift diffusion for calcium dynamics. Our results are shown to be in agreement with reported calcium release data. A modest increase (rough doubling) of the cellular calcium is predicted in the absence of extra-cellular calcium. In particular, the applied field of $15 \mathrm{kV} / \mathrm{cm}$ with $60 \mathrm{~ns}$ pulse duration makes for a strong comparison. No oscillations are predicted and the net recovery period of about $5 \mathrm{~min}$ are both in agreement with published experimental results. A quantitative explanation for the lack of such oscillatory behavior, based on the density dependent calcium fluxes, is also provided.
\end{abstract}

DOI: 10.1103/PhysRevE.75.041920

PACS number(s): 87.16.Ac, 87.16.Dg, 87.16.Xa, 87.16.Gj

\section{INTRODUCTION}

The use of very high electric fields $(\sim 100 \mathrm{kV} / \mathrm{cm}$ or higher) with pulse durations in the nanosecond range has been a very recent development in bioelectrics [1-3]. These nanosecond pulsed electric fields have the following characteristics that could be very useful to some applications: (i) Negligible thermal heating; (ii) the ability to develop large electric fields and peak powers, with a lower energy input; (iii) the capacity to create large transmembrane potentials across subcellular organelles, such as the endoplasmic reticulum; (iv) trigger the activation of various caspases, or change the permeability of voltage-dependent anionic channels (VDACs) and bring about apoptotic cell deaths. Thus deliberate and targeted apoptotic cell killing (e.g., of tumor cells) can potentially be achieved through the use of ultrashort, high-intensity electrical pulses.

A number of other cellular responses have been observed that include the following.

(i) Phosphatidylserine (PS) externalization for average nanosecond pulsed electric fields above $2 \mathrm{MV} / \mathrm{m}[4,5]$. A distinct polarity effect has also been observed, with the externalization predominantly occurring at the anode end. These PS movements and polarity dependences have recently been modeled based on molecular dynamics $[6,7]$. Such electric-field-induced PS externalization can possibly trigger cell death in vivo through macrophage action.

(ii) Cellular apoptosis $[8,9]$ with the behavior depending on pulse duration. Cells subjected to various external electric fields at a constant energy level showed strongest apoptosis markers for the longer $(\sim 300 \mathrm{~ns})$ pulses, less at the shorter ( $\sim 60 \mathrm{~ns})$ durations, and almost negligible effects for an ultrashort $10 \mathrm{~ns}$ pulse [9]. A model based on an energylandscape approach [10] yielded results that were qualitatively in agreement with these observations.

(iii) Calcium release has been observed from the endoplasmic reticulum (ER) in response to external voltage pulses [11-15], with many of the experiments being conducted in the absence of extracellular calcium. External voltage triggering of $\mathrm{Ca}^{2+}$ release could be a vital element in artificially induced cell signaling. This aspect of electric field pulsing has not yet, to the best of our knowledge, been analyzed quantitatively. Since intracellular $\mathrm{Ca}^{2+}$ release leads to some uptake by the mitochondria, such voltage-triggered $\mathrm{Ca}$ release could potentially have secondary effects on the mitochondria. Alterations in the mitochondrial membrane potential due to cationic entry is a possibility. Such transmembrane voltage changes are known to trigger opening of the permeability transition pore $[16,17]$, leading to cytochrome $c$ release and cellular apoptosis.

It has been shown that ultrashort (nanosecond) electric pulses with duration smaller than the plasma membrane charging times have the capacity to permeate into the cell interior and affect subcellular organelles $[1,14,18]$. Arguably, such pulses could affect the mitochondria, in addition to the ER. However, early electrical analysis by Schwan [19] has clearly shown that the transmembrane voltages developed are directly proportional to organelle size. Hence, for a given electric field, it is harder to electroporate smaller structures such as the mitochondria since the requisite threshold voltage is nearly fixed at $\sim 1 \mathrm{~V}$. In recent nanosecond experiments, electric fields as low as $15 \mathrm{kV} / \mathrm{cm}$ have been shown to cause calcium release from the ER. Thus direct experimental evidence for suborganelle effects exists. Possible field-induced effects on mitochondria (e.g., electroporation) were neglected due to the low electric field, nanosecond pulses considered here. In addition, the double walled membrane structure of the mitochondria makes it less likely for electroporation and calcium transport during these shortduration pulses of relatively low $(15 \mathrm{kV} / \mathrm{cm})$ intensity. Finally, stronger effects can be expected on anions (rather than cations) via the VDAC pathways. Our decision to neglect other subcellular organelles was also guided in part by experimental data. Since data from such nanosecond pulsing 
experiments is available for the ER, and our final results seem to match the experimental data fairly well (as shown later), focusing on the ER was deemed to be a reasonable first approach.

An important goal of this contribution is to obtain a quantitative dynamical analysis of the electrically stimulated calcium release from the ER stores by an externally applied nanosecond voltage pulse. Such calcium release plays an important role in several cellular events $[20,21]$ and is thought to initiate factors in the apoptotic pathway [21]. The primary mechanism and its quantification for electrically driven calcium release has not been probed, though electroporation of the ER membrane is conjectured to be a likely pathway.

\section{MODELING DETAILS}

\section{A. Transmembrane field calculations}

Our calcium model requires calculation of the timedependent, transmembrane electric fields and the poration process that facilitates $\mathrm{Ca}^{2}$ ion flux from the ER. It is also critical to determine whether electroporation might occur at the ER, and the time required for such a potential event. An approach to calculating the dynamic electric fields and transmembrane potentials is through a time-domain nodal analysis based on a distributed equivalent circuit representation of a cell and its membrane structures. Details of this method and its implementation have been given by our group elsewhere $[7,10,22]$. Hence only a brief outline will be discussed here. Essentially, the entire volume can be broken up into finite segments, with each segment represented by a parallel resistor-capacitor combination to account for the electrical current flow and charging effects. The computational region considered here was a sector of a sphere that included the cell, the surrounding suspension medium, and part of the ER as shown in Fig. 1. The ER center was taken to be the $r=0$ point, while the intersection of the radial ray with the plasma membrane was the other boundary. The externally applied electric field was taken to be along the axis of symmetry for this spherical problem. Azimuthal symmetry was invoked to eliminate the third dimension. In Fig. 1, some extracellular aqueous medium has been shown. The plasma membrane $(\mathrm{PM})$ is denoted as the "outer membrane." The "inner membrane" represents the ER membrane. The model also included cytosol between these two membranes, as well as the region within the ER. The outer circular segment defines the overall simulation region boundary. In our simulations the total radial distance from the center to the PM boundary was $2.1 \mu \mathrm{m}$, while the ER width was taken to be $0.35 \mu \mathrm{m}$. For accuracy the full quarter of the cell was modeled, though only the on-axis electric fields and potentials were considered for the Ca dynamics.

Simple nodal analysis of circuit theory was applied at each grid point to obtain the time-dependent node voltages in response to the external electric pulse $E_{0}(t)$. Potentials on each node are easily updated at every time step. These voltage values at the ER and plasma membranes of Fig. 2 were fed to a Smoluchowski equation for pore dynamics $[10,22]$. This yielded the time-dependent pore density and distribution of pore area at every membrane grid point. It also effec-

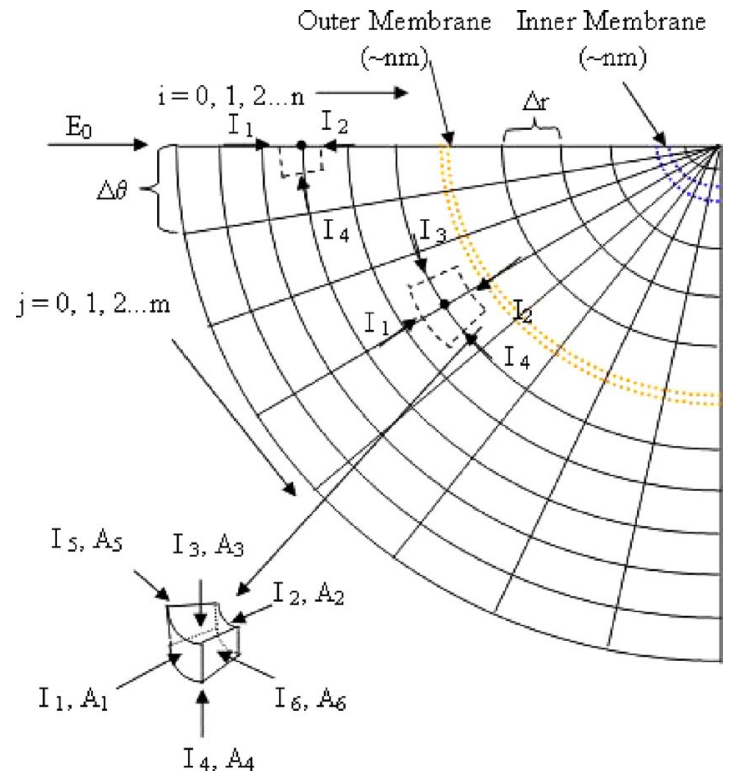

FIG. 1. (Color online) Schematic of one-quarter of the model used to represent a cell for the distributed electrical calculations. The dotted box shows a typical element with current flows. The plasma (outer) membrane and ER (inner) membrane are both sketched.

tively produced a dynamic membrane conductance taking electroporation into account, and yielded drift and diffusive fluxes through the nanopores. The maximal effects are to be expected along the polar axis, and hence only the values at the membrane grid point intersecting the longitudinal electric field were used for consideration of ER poration. This provided the leak flux function discussed in Sec. II C, based on time-dependent pore growth.

\section{B. Background}

Before discussing our calcium model for dynamical concentration changes in both the cytosol and the ER, it is per-

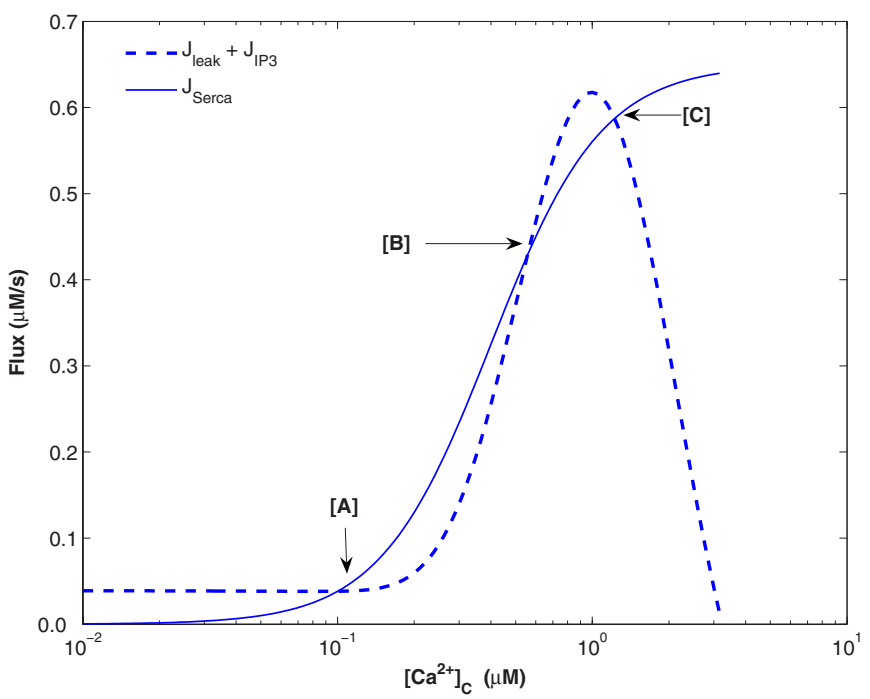

FIG. 2. (Color online) Qualitative sketch of the calcium release and pumping rates as a function of the calcium concentration in the cytosol. 
haps useful to briefly review the calcium signaling and release mechanisms. Intracellular $\mathrm{Ca}^{2+}$ dynamics has the unique feature of facilitating the generation of global events (often of a periodic nature) from local, thermally activated stochastic opening or closing of channels on the ER membrane $[20,21,23,24]$. Such channels are typically closely packed into clusters, called focal sites [25-27] with a random spatial distribution and an average spacing in the 2-6 $\mu \mathrm{m}$ range. A typical channel present in the ER of many cells that facilitates calcium movement is the inositol 1,4,5triphosphate receptor (IP3R) channel. Ryanodine receptors (RyR), though present, are more important in muscle cells [28]. The IP3R channel has an activating binding site for the messenger molecule IP3 ( $m$-gate), an activating site for $\mathrm{Ca}^{2+}$ ( $n$-gate), and an inhibiting $\mathrm{Ca}^{2+}$ binding site ( $h$-gate). Experimental findings suggest that the channel is open if both $\mathrm{Ca}^{2+}$ and IP3 are bound to the activating sites, and at the same time $\mathrm{Ca}^{2+}$ is not bound to the inhibiting site. Binding of $\mathrm{Ca}^{2+}$ to the inhibiting site of one of these subunits closes the channel.

This "open channel" probability increases nonlinearly with the IP3 and calcium concentrations. Hence, any $\mathrm{Ca}^{2+}$ released by one channel increases the open probability of neighboring channels. This provides a self-amplifying, positive-feedback nonlinear mechanism [29] referred to as "calcium-induced calcium release (CICR)." Very high $\mathrm{Ca}^{2+}$ concentrations inhibit the channels. The $\mathrm{Ca}^{2+}$ SERCA pumps remove $\mathrm{Ca}^{2+}$ from the intracellular space. This is necessary since elevated concentrations of $\mathrm{Ca}^{2+}$ are toxic for the cell.

Initial simulation efforts to quantify the intracellular $\mathrm{Ca}^{2+}$ dynamics primarily focused on deterministic continuum models [30-36]. These reaction-diffusion models were able to explain the observed wave patterns, oscillatory, or bistable phenomena. Extensions to these models that allowed the channels to act as discrete $\mathrm{Ca}^{2+}$ sources facilitated the transition from localized to traveling structures [37]. The stochastic behavior seen in spark and puff formation and the rather small number of channels creating a localized event motivated the introduction of stochastic models [38-44]. Mesoscopic aspects of the calcium-release phenomena were probed by Shuai and Jung $[41,42]$. They found that site clustering could effectively allow for collectively enhanced, coherent calcium responses to signals. Homogeneously distributed channels, on the other hand, would not be capable of producing the same large response.

Discrete models have successfully predicted oscillatory dynamical regimes and random, collective calcium enhancements. However, such localized, discrete stochastic models are perhaps not necessary in the present context of fieldassisted calcium release for a variety of reasons. First, it has become apparent based on improved spatial imaging [26,45] that the overall calcium release varies in a continuous fashion despite the stochastic variations in the numbers of individual channels recruited for release and the durations of their openings. Thus, macroscopically, a continuum model remains relevant. Furthermore, the present focus is on very high $(>15 \mathrm{kV} / \mathrm{cm})$ electric fields. This external stimulus produces very strong electrostatic driving forces and gives rise to highly nonequilibrium conditions. Hence it can safely be assumed that all of the channels are effectively driven into the calcium release (or "open") state by the strong external electric signal. Statistical variability can be expected to be minimal, and that all of the discrete sites would be collectively forced past the calcium-release threshold.

\section{Calcium model analyses}

Calcium release from the ER predominantly occurs through IP3R sites [41]. For example, the Xenopus oocyte primarily has IP3 channels. Ryanodine receptors are more important in muscle cells [28]. It may be mentioned that some open issues regarding the RyR mathematical models remain. Hence for simplicity, we have focused here on the IP3R sites as the primary calcium release channel. For other applications, such as cardiac fibrillation that are thought to be coupled to calcium signaling in the ventricles, the RyR channels would need to be included. Our study utilizes the Li-Rinzel two-variable simplification [31] of the De YoungKeizer model [30] with appropriate modifications to account for electric field effects. The basic model has been used in the past (but without any external electric stimulation) for various analyses $[29,46]$. According to this model, calcium flux between the ER and the intracellular space is driven by the following three processes: (i) $\mathrm{Ca}^{2+}$ outflow mediated by the IP3 channel, (ii) a small diffusion-driven $\mathrm{Ca}^{2+}$ leakage from the ER into the cytosol, and (iii) the SERCA ATPase pumps that drive $\mathrm{Ca}^{2+}$ back into the ER to maintain the resting (basal) calcium levels.

Here we include the additional mechanism of electric field driven $\mathrm{Ca}^{2+}$ outflow from the ER to the cytosol. Electroporation of the ER membrane, coupled with the electrical driving force on the calcium cations contributes to this outflow. In a sense, the ER becomes more "leaky." This effect has been included in our one-dimensional, time-dependent reaction-diffusion model through a time-dependent leak-flux term. The equations for $\mathrm{Ca}^{2+}(z, t)$ density changes in the cytosol and ER are expressed, in general, by the continuity equation as

$$
\partial\left\{\left[\mathrm{Ca}^{2+}\right]\right\} / \partial t=-\partial F(z, t) / \partial z+G(z, t)-R(z, t),
$$

where $F(z, t)$ is the passive calcium flux at any location " $z$ " and time " $t$," while $G(z, t)$ and $R(z, t)$ are possible $\left[\mathrm{Ca}^{2+}\right]$ generation and recombination (or attachment) rates that could include pumps, channels, and pores. In our treatment, generation, recombination or attachment of $\mathrm{Ca}^{+2}$ ions, action of pumps, channels, pores, etc. has been ignored within the bulk and only taken into account at the ER-cytosol boundary. The passive flow of $\left[\mathrm{Ca}^{2+}\right]$ ions can be expressed in terms of drift and diffusive processes. Assuming that the drift velocity of the $\left[\mathrm{Ca}^{2+}\right]$ ions in response to the highest local electric fields is much smaller than their thermal velocities, and that the role of scattering can be approximated by a relaxation time

" $\tau$," the flux $F(z, t)$ can be cast into the following "driftdiffusion" form:

$$
F(z, t)=\left[\mathrm{Ca}^{2+}\right] \mu E(z, t)-D(z, t)\left(\partial\left[\mathrm{Ca}^{2+}\right] / \partial z\right),
$$

where $\mu$ is the ion mobility, $E(z, t)$ is the space- and timedependent local electric field, and $D$ the diffusion coefficient. 
These parameters are simply related to the relaxation time " $\tau$ " as $\mu=q\left\langle v^{2} \tau\right\rangle /\left(3 k_{B} T\right)$, and $D=\left(k_{B} T / q\right) \mu$, where $q$ is the elementary charge, $T$ the temperature, $k_{B}$ the Boltzmann constant, and $v$ the individual ionic velocity, while \langle\rangle denotes an ensemble average over $\mathrm{Ca}^{2+}$ ions.

For completeness, it may be pointed out that there are some approximations inherent in our use of the above driftdiffusion scheme. For example, the diffusion coefficient and mobility are taken as fixed, invariant parameters. Strictly, this can only be done if the system is stationary, not far from equilibrium, and strong local inhomogeneities do not exist. For nonstationary, non-Markovian processes, the diffusion coefficient needs to be evaluated as an integral over a twotime velocity autocorrelation function [46]. Due to the ultrashort electric pulse, we assumed near stationarity. Treatments of time variations of transport parameters in an aqueous medium over time scales of nanoseconds or shorter, and in the presence of external electric fields, have been reported by our group elsewhere [47]. Also, since the $\left[\mathrm{Ca}^{2+}\right]$ release and other biochemical changes are relatively perturbative in nature with almost no impact or deviations in overall internal scattering rates, the $\mu$ and $D$ transport parameters can essentially be assumed homogeneous. Thus using Eq. (2) in Eq. (1) yield

$$
\begin{aligned}
\partial\left\{\left[\mathrm{Ca}^{2+}\right]\right\} / \partial t= & D(z, t)\left(\partial^{2}\left[\mathrm{Ca}^{2+}\right] / \partial z^{2}\right)-\mu \partial\left\{\left[\mathrm{Ca}^{2+}\right] E(z, t)\right\} / \partial z \\
& +G(z, t)-R(z, t) .
\end{aligned}
$$

Since an analytical solution cannot be obtained for the above equation, a numerical approach was used based on a uniform discretization in space (spacing " $d z$ ") and time (interval " $d t$ "). The details are briefly given next for completeness. The entire simulation region was divided into a set of " $N$ " uniformly sized boxes of thickness " $d z$." In this discretized representation, index " $i$ " denotes the $i$ th time step $(t=\{i-1\} d t)$, and " $j$ " denotes the $j$ th spatial step $(z=\{j$ $-1\} d z)$. Thus a total of $(N+1)$ grid points were chosen. Voltages were calculated at all of the $(N+1)$ grid points, while the $\mathrm{Ca}^{2+}$ concentration and electric field were defined at the center of each box (i.e., between adjacent grid points.) The indices $j=(J-1)$ and $j=J$ represent the two boxes on either side of the ER-cytosol boundary. The results of discretizing Eq. (3) are given below for various regions within the simulation zone.

The governing transport equation in the outer cytosol region can be expressed as

$$
\begin{aligned}
& \left\{\mathrm{Ca}^{2+}{ }_{i+1, j}-\mathrm{Ca}^{2+}{ }_{i, j}\right\} /(d t) \\
& =D_{c}\left\{\mathrm{Ca}^{2+}{ }_{i, j+1}-2 \mathrm{Ca}^{2+}{ }_{i, j}+\mathrm{Ca}^{2+}{ }_{i, j-1}\right\} /(d z)^{2} \\
& \\
& \quad-\mu_{c}\left[\left\{\mathrm{Ca}^{2+}{ }_{i, j+1} E_{i, j+1}\right\}-\left\{\mathrm{Ca}^{2+}{ }_{i, j-1} E_{i, j-1}\right\}\right] /(2 d z) \\
& \quad \text { for } J<j<N,
\end{aligned}
$$

where $\mathrm{Ca}^{2+}{ }_{i, j}$ denotes the calcium ion concentration $\left[\mathrm{Ca}^{2+}\right]$ at the $i$ th time step and $E_{i, j}$ similarly denotes the value of the discretized electric field. In Eq. (4a), $D_{c}$ and $\mu_{c}$ denote the diffusion coefficient and mobility values in the cytosol. At the last box next to the plasma membrane boundary $(j=N)$, the governing transport equation is

$$
\begin{aligned}
& \left\{\mathrm{Ca}^{2+}{ }_{i+1, j}-\mathrm{Ca}^{2+}{ }_{i, j}\right\} /(d t) \\
& =-D_{c}\left\{\mathrm{Ca}^{2+}{ }_{i, j}-\mathrm{Ca}^{2+}{ }_{i, j-1}\right\} /(d z)^{2}+\mu_{c}\left[\left\{\mathrm{Ca}^{2+}{ }_{i, j} E_{i, j}\right\}\right. \\
& \left.\quad+\left\{\mathrm{Ca}^{2+}{ }_{i, j-1} E_{i, j-1}\right\}\right] /(2 d z) \text { for } j=N .
\end{aligned}
$$

Similarly, the discretized continuity equation in the inner ER region can be expressed as

$$
\begin{aligned}
& \left\{\mathrm{Ca}^{2+}{ }_{i+1, j}-\mathrm{Ca}^{2+}{ }_{i, j}\right\} /(d t) \\
& =D_{E}\left\{\mathrm{Ca}^{2+}{ }_{i, j+1}-2 \mathrm{Ca}^{2+}{ }_{i, j}+\mathrm{Ca}^{2+}{ }_{i, j-1}\right\} /(d z)^{2} \\
& \quad-\mu_{E}\left[\left\{\mathrm{Ca}^{2+}{ }_{i, j+1} E_{i, j+1}\right\}-\left\{\mathrm{Ca}^{2+}{ }_{i, j-1} E_{i, j-1}\right\}\right] /(2 d z) \\
& \quad \text { for } 1<j<(J-1),
\end{aligned}
$$

where $E_{E}$ and $\mu_{E}$ denote the diffusion coefficient and mobility values in the ER. At the $j=1$ grid point (corresponding to the first box within the ER), the discretized equation is

$$
\begin{aligned}
& \left\{\mathrm{Ca}^{2+}{ }_{i+1, j}-\mathrm{Ca}^{2+}{ }_{i, j}\right\} /(d t) \\
& =D_{E}\left\{\mathrm{Ca}^{2+}{ }_{i, j+1}-\mathrm{Ca}^{2+}{ }_{i, j}\right\} /(d z)^{2}-\mu_{E}\left[\left\{\mathrm{Ca}^{2+}{ }_{i, j} E_{i, j}\right\}\right. \\
& \left.\quad+\left\{\mathrm{Ca}^{2+}{ }_{i, j+1} E_{i, j+1}\right\}\right] /(2 d z) \text { for } j=1 .
\end{aligned}
$$

For $j=J+1$ (cytosol box just to the right of the ER boundary), the continuity equation becomes

$$
\begin{aligned}
\left\{\mathrm{Ca}^{2+}{ }_{i+1, j}-\mathrm{Ca}^{2+}{ }_{i, j}\right\} /(d t) & \\
= & D_{c}\left\{\mathrm{Ca}^{2+}{ }_{i, j+1}-\mathrm{Ca}^{2+}{ }_{i, j}\right\} /(d z)^{2}+g(t)-\mu_{c}\left[\left\{\mathrm{Ca}^{2+}{ }_{i, j+1} E_{i, j+1}\right\}\right. \\
& \left.+\left\{\mathrm{Ca}^{2+}{ }_{i, j} E_{i, j}\right\}\right] /(2 d z)+\left[J_{\text {IP3R }}-J_{\text {SERCA }}+J_{\text {leak }}\right] / F_{C},
\end{aligned}
$$

where $g(t)$ denotes a poration dependent "leak flux" function. In Eq. $(4 \mathrm{e}), j_{\mathrm{IP} 3 \mathrm{R}}, j_{\mathrm{SERCA}}$, and $j_{\text {leak }}$ denote the usual flux terms at the ER boundary, while $F_{C}$ is the volume fraction of the cytosol relative to the total cell volume. Similarly, for $j=(J$ $-1)$, one has

$$
\begin{aligned}
\left\{\mathrm{Ca}^{2+}{ }_{i+1, j}-\mathrm{Ca}^{2+}{ }_{i, j}\right\} /(d t) & \\
=- & D_{E}\left\{\mathrm{Ca}^{2+}{ }_{i, j}-\mathrm{Ca}^{2+}{ }_{i, j-1}\right\} /(d z)^{2}-g(t)+\mu_{E}\left[\left\{\mathrm{Ca}^{2+}{ }_{i, j} E_{i, j}\right\}\right. \\
& \left.+\left\{\mathrm{Ca}^{2+}{ }_{i, j-1} E_{i, j-1}\right\}\right] /(2 d z)-\left[J_{\text {IP3R }}-J_{\text {SERCA }}+J_{\text {leak }}\right] / F_{E},
\end{aligned}
$$

where $F_{E}$ is the volume fraction of the ER relative to the total cell volume (i.e., $F_{E}+F_{C}=1$ ).

Initial starting concentrations were set on the basis of $E_{i, j}=0$ and $\left[j_{\mathrm{IP} 3 \mathrm{R}}-j_{\mathrm{SERCA}}+j_{\text {leak }}\right]=0 . \mathrm{A} \mathrm{Ca}^{2+}$ value of $0.1 \mu M$ was chosen inside the cytosol (i.e., $\mathrm{Ca}^{2+}{ }_{i=0, j}=0.1 \mu M$ for $j$ $\leqslant j \leqslant N$ ), while the concentration inside the ER ( was obtained from the initial steady-state condition of $\left[j_{\text {IP3R }}-j_{\text {SERCA }}+j_{\text {leak }}\right]=0$. Thus $\mathrm{Ca}^{2+}{ }_{i=0, j}=\mathrm{Ca}^{2+}{ }_{\text {ER } 0}$ for $1 \leqslant j$ $\leqslant(J-1)$.

The discretized value of the electric field $E_{i, j}\{=E(z, t)\}$ is dictated by the duration of the externally applied voltage stimulation. The requisite internal electric fields were derived at each time instant from the distributed electric representation shown in Fig. 1. Upon termination of the external voltage, the $E_{i, j}$ term quickly falls to zero at all grid points in the simulation region. Beyond such times, only the diffusion term is operative for $\mathrm{Ca}^{2+}$ transport. The "flux leak function" 
$g(t)$ accounts for the ER membrane poration process, and includes the finite delay in its creation in response to the external voltage pulse. It is obtained as an input parameter from calculations of membrane poration based on the Smoluchowski equation, discussed in the preceding section. Thus the function $g(t)$ models time dependent "poration" and $\mathrm{Ca}^{2+}$ outflow from the ER at the ER-cytosol interface. In the scenario simulated here, the external voltage pulse is sufficiently large to porate the ER before its termination. Hence, during the later part of the voltage pulse (nanosecond regime), we have in effect a source term continually feeding $\mathrm{Ca}^{2+}$ into the cytosol, together with the drift of the $\mathrm{Ca}^{2+}$ ions away in the direction of the plasma membrane.

It may be mentioned that the above numerical implementation is based on an explicit scheme. An implicit scheme is easily realized by replacing terms such as $\mathrm{Ca}^{2+}{ }_{i, j}$ on the right side of Eqs. (4a)-(4f) by $\left[\left(\mathrm{Ca}^{2+}{ }_{i, j}+\mathrm{Ca}^{2+}{ }_{i+1, j}\right) / 2\right]$. This results in a set of coupled simultaneous equations that can be cast in a matrix form and solved to yield the relevant concentrations at the succeeding time steps. The implicit scheme has the advantage of greater numerical stability. In our case, the implicit scheme was implemented as well, and solution to the system of equations obtained through LU decomposition of the matrix. The difference between the two solutions was negligible, and there were no stability issues with the explicit scheme for the grid sizes chosen.

The mathematical expressions used for the various fluxes at the ER boundary [for example, indicated in Eqs. (4e) and (4f)] are given next. A similar discretized numerical implementation was used and, for simplicity, only the algebraic equations are given below. The constituent current densities $j_{\mathrm{IP3R}}, j_{\mathrm{SERCA}}$, and $j_{\text {leak }}$ can be expressed [48] as

$$
\begin{gathered}
J_{\text {IP } 3 \mathrm{R}}=V_{\text {IP } 3 \mathrm{R}} m(t)^{3} h(t)^{3}\left(\left[\mathrm{Ca}^{2+}\right]_{E}-\left[\mathrm{Ca}^{2+}\right]_{C}\right), \\
J_{\text {leak }}=V_{\text {leak }}\left(\left[\mathrm{Ca}^{2+}\right]_{E}-\left[\mathrm{Ca}^{2+}\right]_{C}\right), \\
J_{\text {SERCA }}=V_{\text {SERCA }}\left(\left[\mathrm{Ca}^{2+}\right]_{C}\right)^{2} /\left\{\left(K_{\text {SERCA }}\right)^{2}+\left(\left[\mathrm{Ca}^{2+}\right]_{C}\right)^{2}\right\} .
\end{gathered}
$$

In the above formulation, $V_{\text {IP3R }}, V_{\text {leak }}$, and $V_{\text {SERCA }}$ are constants that have been reported in the literature. The variables $m(t)$ and $h(t)$ are time-dependent, and given by the following expressions:

$$
m(t)=\left(\left[\mathrm{IP}_{3}\right] /\left[\mathrm{IP}_{3}+K_{\mathrm{IP} 3}\right]\right)\left\{\left[\mathrm{Ca}^{2+}\right]_{C} /\left(\left[\mathrm{Ca}^{2+}\right]_{C}+K_{\mathrm{act}}\right)\right\},
$$

and

$$
d h(t) / d t=\left[h_{\infty}-h(t)\right] / \tau,
$$

where

$$
h_{\infty}=K_{\mathrm{inh}} /\left\{\left(K_{\mathrm{inh}}+\left[\mathrm{Ca}^{2+}\right]_{C}\right)\right\} .
$$

In Eqs. (6a)-(6c), $K_{\text {act }}$ (and $K_{\text {inh }}$ ) are the activating (and inhibiting) $\mathrm{Ca}^{2+}$ binding site dissociation constants, $\left[\mathrm{IP}_{3}\right]$ is

\begin{tabular}{|c|c|}
\hline Model parameter & Value \\
\hline$K_{\mathrm{IP} 3}$ & $0.15 \mu M$ \\
\hline$K_{\text {act }}$ & $0.8 \mu M$ \\
\hline$V_{\mathrm{IP} 3 \mathrm{R}}$ & $8.5 \mathrm{~s}^{-1}$ \\
\hline$V_{\text {leak }}$ & $0.01 \mathrm{~s}^{-1}$ \\
\hline$V_{\text {SERCA }}$ & $2.6 \mu \mathrm{M} / \mathrm{s}$ \\
\hline$K_{\text {SERCA }}$ & $0.2 \mu M$ \\
\hline$V_{\text {prod }}$ & $0.075 \mu M / s$ \\
\hline$K_{\text {prod }}$ & $0.4 \mu M$ \\
\hline$V_{1}$ & $0.001 \mu M / \mathrm{s}$ \\
\hline$V_{2}$ & $0.005 \mu \mathrm{M} / \mathrm{s}$ \\
\hline$V_{3}$ & $0.02 \mu \mathrm{M} / \mathrm{s}$ \\
\hline$D_{C}$ & $16 \mu \mathrm{m}^{2} / \mathrm{s}$ \\
\hline$D_{E}$ & $16 \mu \mathrm{m}^{2} / \mathrm{s}$ \\
\hline$D_{\mathrm{IP} 3}$ & $283 \mu \mathrm{m}^{2} / \mathrm{s}$ \\
\hline$K_{\mathrm{inh}}$ & $1.9 \mu M$ \\
\hline$\tau$ & $2 \mathrm{~s}$ \\
\hline$\lambda$ & 30.0 \\
\hline$F_{C}$ & 0.8 \\
\hline$F_{E}$ & 0.2 \\
\hline
\end{tabular}
the time-dependent, spatially variable concentration of IP3 in the cytosol, while $\tau$ is the inactivation time constant. Values of the various constants and parameters are generally well established in the literature, and here many of those used by
TABLE I. Parameters for the Ca model simulations.

Wagner et al. [48] have been implemented. For completeness, the parameter set used in our simulations is given in Table I.

The complete dynamics requires inclusion of IP3 production and its degradation. Basically, $\mathrm{Ca}^{2+}$-induced IP3 production at the ER and plasma membranes is given by the following relation:

$$
J_{\text {IP3_prod }}=V_{\text {prod }}\left(\left[\mathrm{Ca}^{2+}\right]_{C}\right)^{2} /\left\{\left(\left[\mathrm{Ca}^{2+}\right]_{C}\right)^{2}+K_{\text {prod }}^{2}\right\},
$$

with appropriate constants $V_{\text {prod }}$ and $K_{\text {prod. }}$. The IP3 degradation model of Wagner et al. [48] is based on IP3 hydrolysis by 5-phosphatase to inositol-1,4-bisphosphate (IP2) and the phosphorylation by 3-kinase to inositol-1,3,4,4tetrakisphosphate (IP4). The corresponding rates, $j_{\text {phos }}$ and $j_{\text {kinase }}$, are given as

$$
J_{\text {phos }}=V_{3}[\mathrm{IP} 3] /([\mathrm{IP} 3]+30),
$$

$$
\begin{aligned}
J_{\text {kinase }}= & (1-\theta) V_{1}[\mathrm{IP} 3] /([\mathrm{IP} 3]+2.5)+\theta V_{2}[\mathrm{IP} 3] /([\mathrm{IP} 3] \\
& +0.5),
\end{aligned}
$$

where $V_{1}, \quad V_{2}, \quad$ and $V_{3}$ are constants, and $\theta$ $=\left[\mathrm{Ca}^{2+}\right]_{C} /\left(\left[\mathrm{Ca}^{2+}\right]_{C}+0.39\right)$. The concentrations [IP3], $\left[\mathrm{Ca}^{2+}\right]_{C}$, and $\left[\mathrm{Ca}^{2+}\right]_{E}$ are all in micromolar units. The dynamical evolution of the IP3 concentration is then given in terms of the various creation-annihilation rates and the flux as

$$
\partial[\mathrm{IP} 3] / \partial t=D_{\mathrm{IP} 3} \partial^{2}[\mathrm{IP} 3] / \partial^{2} x+\left[J_{\mathrm{IP} 3 \text { _prod }}-\lambda\left(J_{\text {kinase }}-J_{\text {phos }}\right)\right] / F_{C},
$$

with $\lambda$ being the IP3 rate scaling factor. 
It may be mentioned that theoretically, besides the ionic drift, diffusion through the electrically created nanopores can also occur. However, such calcium diffusive fluxes can be expected to be relatively negligible for the following reasons. (i) Discontinuities in permittivity between the ER membrane and its surrounding aqueous medium impede ionic transport in the vicinity of a nanopore. Such small-area nanopores (radii $\sim 1 \mathrm{~nm}$ or less) within lipid membranes have been predicted based on continuum approaches [49] and also on the basis of molecular dynamics simulations [6,7]. Their small diameters result from the ultrashort time scales during which the external driving fields are applied, thereby preventing sustained and large pore expansions. Experimentally, the nanopores have indirectly been detected by using various fluorescent dyes [50]. For nanopores, both the electrostatic interactions and Born solvation energy [51,52] of ions approaching such apertures are strongly enhanced. Hence, in the absence of an electric field (upon termination of the ultrashort voltage pulse, for example), the ionic $\mathrm{Ca}^{2+}$ diffusion into the cytosol through the "nanopipe" would be minimal. (ii) Nanopore resealing can be expected to be fairly rapid after termination of the nanosecond pulse given the small pore size. Very short resealing times (under $5 \mathrm{~ns}$ ) have been reported in the literature [53] based on molecular dynamics simulations. Such resealing would further severely restrict any additional contributions to diffusive leak-out from the ER. For the above reasons, electrically induced $\mathrm{Ca}^{2+}$ transfer from the ER was taken to be the operative via the drift mechanism only during external pulsing.

Some qualitative insights into the $\mathrm{Ca}^{2+}$ release dynamics are provided by the concentration-dependent rates. The $j_{\text {SERCA }}$ function is sigmoidal and increases monotonically with the calcium concentration in the cytoplasm. A qualitative sketch is given in Fig. 2. The values were obtained from Eqs. (5) and (6) on applying the parameters listed in Table I. The calcium release rate exhibits a bell-shaped curve as sketched in Fig. 2. The $j_{\mathrm{IP} 3 \mathrm{R}}$ contribution to release depends on " $m$ " and the differential $\left\{\left[\mathrm{Ca}^{2+}\right]_{E}-\left[\mathrm{Ca}^{2+}\right]_{C}\right\}$. The rate of calcium leakage is relatively very small, and does not have much bearing on the dynamics [54]. These rates, which do not take any account of electrically induced fluxes, underscore the possibility of attaining either bistable or oscillatory operating points. For example, in Fig. 2 the intersections of $j_{\text {SERCA }}$ with $\left\{j_{\text {IP3R }}+j_{\text {leak }}\right\}$ are represented by points " $A$, , " $B$," and " $C$." Of these, " $A$ " and " $C$ " are stable operating points, while " $B$ " denotes an unstable, oscillatory state. Thus it would seem possible to drive the system from an initial state of low cytosolic $\mathrm{Ca}^{2+}$ levels towards relatively high calcium levels by initiating a triggered release.

The primary role of an external voltage is the additional release of $\mathrm{Ca}^{2+}$ due to nanoporation at the ER and the electrically induced drift. This outflow (to first order), would only depend on the calcium concentration within the ER, the voltage magnitude, and pulse duration, and be independent of the $\mathrm{Ca}^{2+}$ concentration in the cell. Effectively, the electrical triggering would shift the calcium release curve vertically, raising the outflow rates for a given $\left[\mathrm{Ca}^{2+}\right]_{C}$ concentration. Two possible scenarios might then result. First, for long voltage pulses, the electrically driven outflow would be relatively long lived, and a high calcium concentration (with

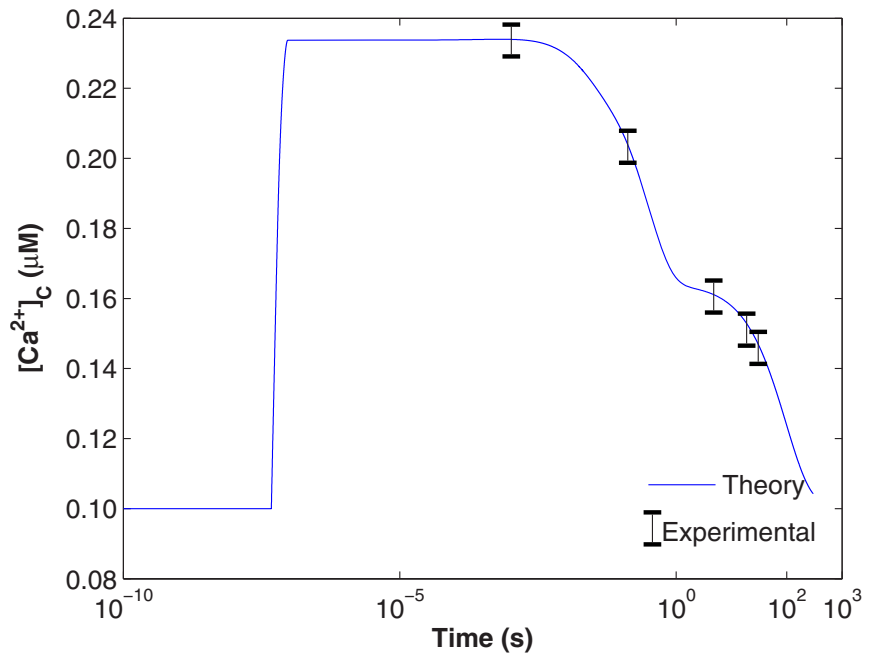

FIG. 3. (Color online) Simulation results for average Ca concentration in cytosol as a function of time. The experimental data points are from the reports by White et al. [15].

additional capacitive entry [55-57] from the extracellular medium) could be attained. However, for short pulse durations, the electrically induced flow would start from an initial higher level (as compared to the equilibrium level), but merge with normal $J_{\text {IP3R }}$ curve at the end of the voltage pulse. This could lead to two intersections with the sigmoidal $J_{\text {SERCA }}$ curve-points " $B$ " and " $C$."

For completeness, it may be pointed out that the above scenario changes slightly in the absence of calcium within the extracellular medium. Such conditions have been created experimentally through the use of chelating agents such as BAPTA, and the resulting calcium dynamics were reported $[8,14,15]$. If capacitive entry through store-operated calcium channels in the plasma membrane [55-57] was inhibited, then the high intracellular $\mathrm{Ca}^{2+}$ levels required for the bistable state " $C$ " would be very difficult to attain.

\section{RESULTS AND DISCUSSION}

Simulations for the time-dependent calcium concentrations within both the cytoplasm and endoplasmic reticulum, in response to a $60 \mathrm{~ns}, 15 \mathrm{kV} / \mathrm{cm}$ electric pulse, were carried out. These electrical pulse parameters were chosen to match previous experimental reports $[12,14,15,58]$ on electric-fieldinduced calcium release. All calculations assumed an absence of extracellular calcium. The results for the averaged $\mathrm{Ca}^{2+}$ concentration in the cytosol are given in Fig. 3. The plot also includes experimental data points that have been reported in the literature [15]. In Fig. 3, the $\mathrm{Ca}^{2+}$ concentration starts from an initial $0.1 \mu M$ value and increases in time to a maximum of about $0.23 \mu \mathrm{M}$. The data is for HL-60 cells. The sharp increase, seen during the initial phase, is due to electric-field-induced poration within the ER that leads to a $\mathrm{Ca}^{2+}$ outflow. Drift rather than diffusion is the primary transport mechanism during this initial phase when the external voltage remains applied. Beyond this initial sharp $\mathrm{Ca}^{2+}$ release, IP3R-activated calcium transfer arising from the CICR mechanism begins to set in. A slow and more gradual 


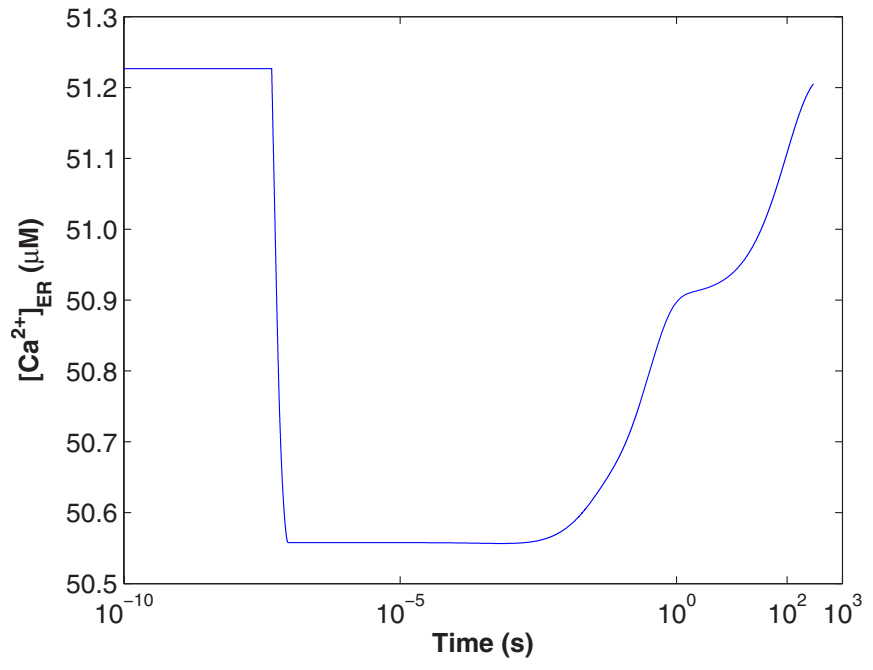

FIG. 4. (Color online) Simulation results for average Ca concentration in the ER as a function of time.

recovery down to the initial concentrations is predicted, with final steady state being achieved after about $300 \mathrm{~s}$. This $\sim 4-5$ min recovery time is in accord with reported measurements $[8,14,15]$ and involves a dynamical interplay between the CICR mechanism, action of the SERCA pumps, and ER membrane leakage. The experimental data of White et al. [15] shown in Fig. 3 matches the predictions well. The model predictions were within the experimental error deviations for all the points. Due to limitations in the temporal resolution of experimental techniques, values of the $\mathrm{Ca}^{2+}$ concentration at the earlier times could not be measured, and hence such data points could not be shown. It is also interesting to note that our simulations demonstrate that very high densities, as needed to attain the bistable state " $C$ " of Fig. 1, cannot be achieved. Hence a long-lived, calcium-loaded state for cells is precluded in situations of extracellular free media. Even the oscillatory state is predicted to be difficult to attain at these relatively modest increases. The experimental data in Ca-free extracellular environments $[8,14,15]$ is in accord with this prediction, with no reports of oscillations.

The changes in the $\mathrm{Ca}^{2+}$ density with time within the ER are shown in Fig. 4. It exhibits an initial rapid decrease due to $\mathrm{Ca}^{2+}$ outflow following field-induced poration. The CICR mechanism continues to cause some $\mathrm{Ca}^{2+}$ outflow, and the density within the ER remains relatively low for a long duration. The overall plot of Fig. 4 roughly exhibits an inverted $\mathrm{U}$ shape. Following initial depletion in the ER, the gradual restoration of the $\mathrm{Ca}^{2+}$ concentrations through SERCA pumping is predicted, after about $5 \mathrm{~min}$.

The simulations also underscore spatiotemporal nonuniformities in calcium concentration caused by E-pulsing. For example, the cytosolic $\mathrm{Ca}^{2+}$ concentration in a region immediately adjacent to the ER is shown in Fig. 5 as a function of time. Though the general shape of the curve is roughly similar to that of Fig. 3, a much stronger localized increase (of up to $6.2 \mu M)$ is predicted with near high levels sustained until about $5 \mu$ s. Electroporation was initiated after about $\sim 48 \mathrm{ns,}$ leading to the strong and direct $\mathrm{Ca}$ release from the ER into the adjacent spatial site through electric field assisted ionic

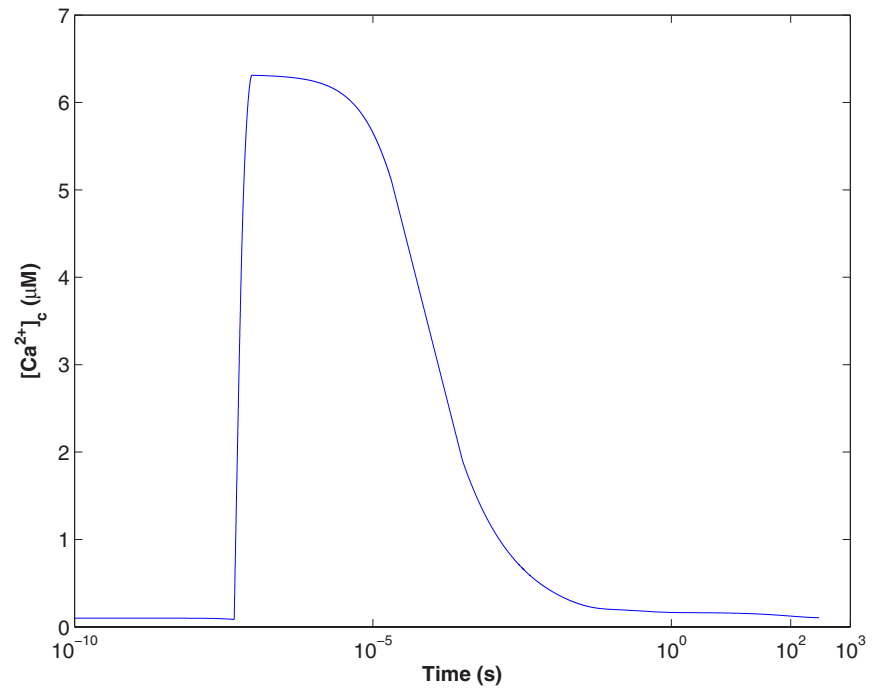

FIG. 5. (Color online) Results for cytosolic Ca concentration near the ER as a function of time.

drift. The concentration increased sharply. After electric field turnoff, the pores slowly close, and $\mathrm{Ca}^{2+}$ diffusion away from this region into the cytosol (towards the plasma membrane) begins. There is an interplay between this diffusive decrease and $\mathrm{Ca}^{2+}$ creation by the CICR mechanism at the IP3 receptors. Hence a fairly long-lived maximum of about $6.2 \mu M$ is sustained until about $5 \mu \mathrm{s}$. At longer times, diffusion and continued flow towards the plasma membrane, higher SERCA pumping rates, and reductions in $J_{\text {IP3 }}$ collectively contribute to the decrease. Experimentally, such spatial inhomegeneities would be difficult to resolve and quantify based on the usual fluorescent intensity data.

The temporal behavior at the last mesh point near the plasma membrane (PM) is somewhat different and is shown in Fig. 6. The initial small rise is due to the electric field assisted calcium ion drift from regions in the cytosol near the PM. This calcium drift leads to a slight "pile-up" at the PM boundary. The applied electric fields were not high enough to

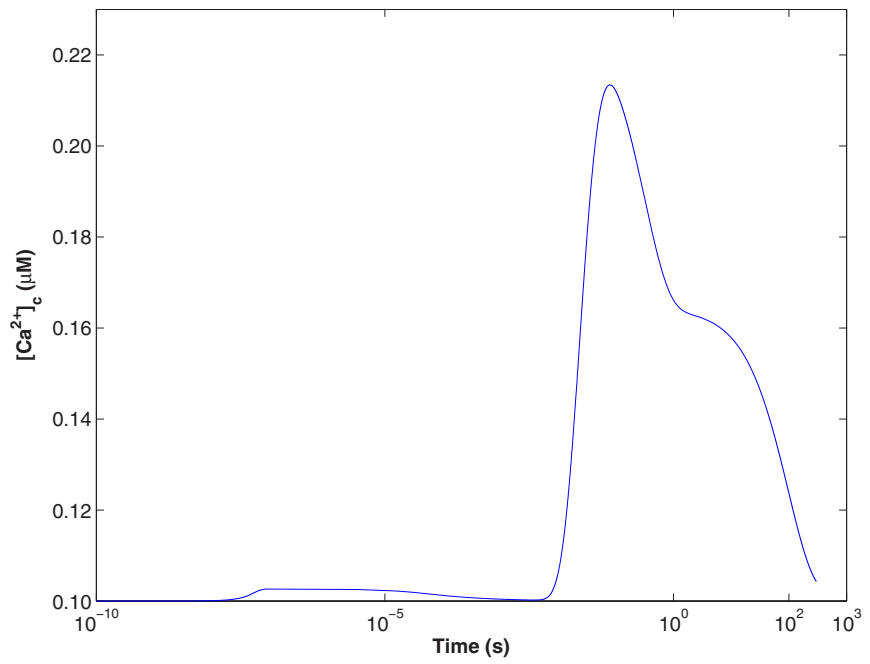

FIG. 6. (Color online) Results for cytosolic Ca concentration near the plasma membrane as a function of time. 


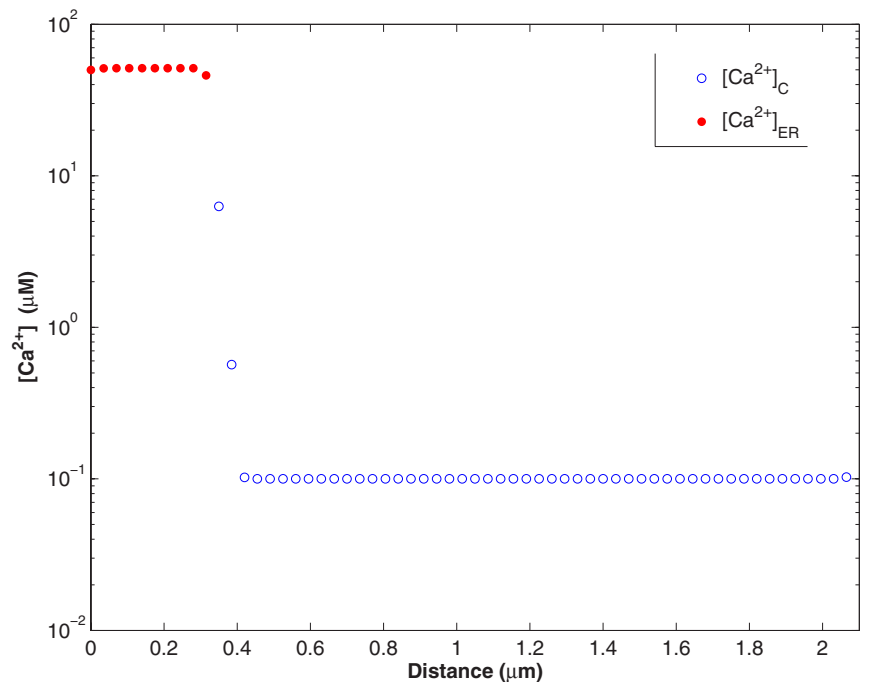

FIG. 7. (Color online) Snapshots of Ca concentration at $90 \mathrm{~ns}$ in the cytosol and the ER.

cause electroporation of the plasma membrane. Hence calcium "leakout" was not operative in the "closed system" modeled here. Also, the absence of extra-cellular calcium precluded possible capacitive inflows. In Fig. 6, the initial buildup at the PM is followed by a gradual diffusive flowback, and hence the concentration decreases in the $0.1-100.0 \mu \mathrm{s}$ time frame. At still later times $(\sim 50 \mathrm{~ms})$, a secondary $\mathrm{Ca}^{2+}$ wave originally emanating from the porated ER release reaches the PM by diffusive transport. The overall result is that the cytosol at the PM boundary experiences a second maxima. In the presence of extracellular calcium, however, one would have expected a primary burst of capacitive entry at roughly $\sim 10 \mu \mathrm{s}$, followed by multiple ripples and wavelets of calcium entry.

Next, a temporal snapshot of field-induced calcium release and the resulting spatial distribution is shown in Fig. 7 at $90 \mathrm{~ns}$ soon after the voltage pulse. Only numerical simulations can provide such detailed insights, since experimental techniques lack the spatial and temporal resolution necessary for such detection. The sudden and large injection of $\mathrm{Ca}^{2+}$ from the ER at the left cytosol boundary (located at $0.3 \mu \mathrm{m}$ ) is obvious. At this relatively early time, most of the injected $\mathrm{Ca}^{2+}$ lies in the neighborhood of the ER. Localized regions of the cytosol next to the ER have relatively high densities, while points further away in the cytosol remain close to their unperturbed densities of $\sim 0.1 \mu M$.

For completeness, time evolution of the $m(t)$ and $h(t)$ functions of Eqs. (6a)-(6c) are shown in Figs. 8 and 9. A large percentage increase in $m(t)$ is predicted in Fig. 8, followed by a near-complete recovery after $300 \mathrm{~s}$. The rapid rise is due to the electroporation, followed by a steady CICR mechanism as calcium levels increase in the localized region adjacent to the ER. Subsequent transport of calcium towards the PM and the cessation of Ca outflow from the ER causes the decrease beyond $10 \mu \mathrm{s}$. The $h(t)$ behavior of Fig. 9 follows directly from Eq. (6b) and is in response to variations in the Ca-levels near the ER side of the cytosol. Finally, the dynamical rates of flux associated with the various transfers

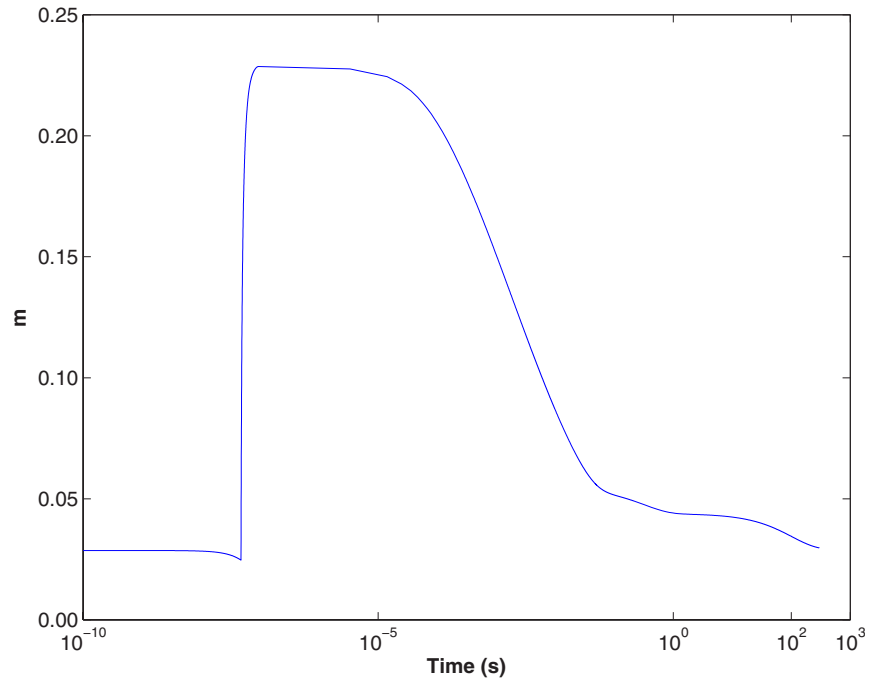

FIG. 8. (Color online) Results for $m(t)$ versus time.

at the ER membrane are shown in Fig. 10. The slight initial decrease in SERCA pump rate (prior to electroporation but with the external field applied) is due to the $\mathrm{Ca}^{2+}$ depletion via ionic drift in the cytosol neighboring the ER boundary. The main influence of $E$ pulsing is the large increase in the SERCA pump rates following Ca release from the ER. The slight $\mathrm{Ca}$ depletion within the ER and the cytosolic accumulation just outside lead to a slight time-dependent decrease in the "leakage" rate at the early times. The IP3 flux exhibits a local maxima until around $50 \mu \mathrm{s}$ and is the direct result of a corresponding increase in $m(t)$. A final steady state is achieved with balance between the outpumping and $\mathrm{Ca}$ recovery rates.

\section{SUMMARIZING CONCLUSIONS}

Calcium modulation is an important signaling process, and could even contribute to cell apoptosis through ionicinduced perturbations in transmembrane potential at the mi-

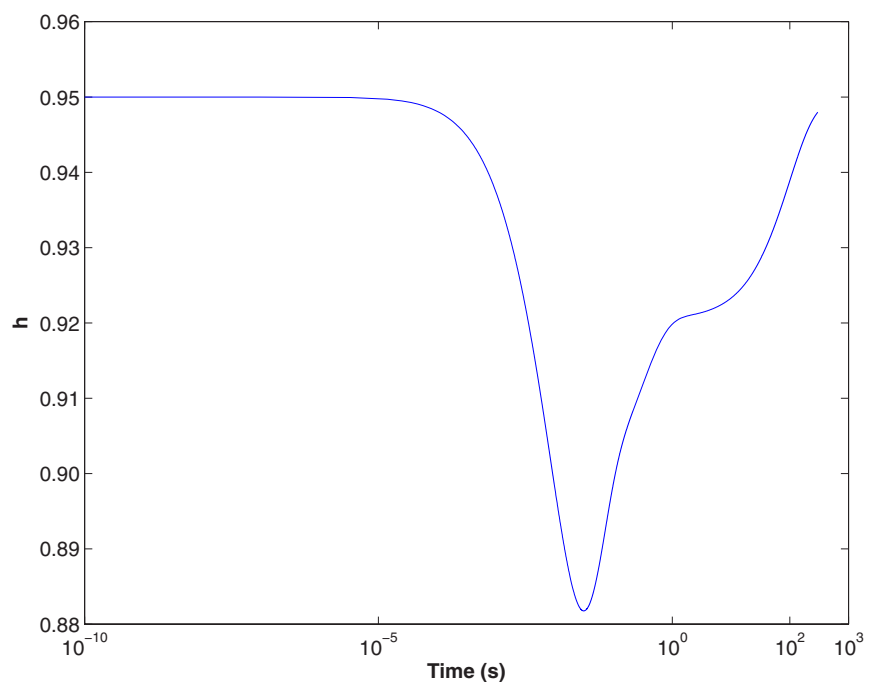

FIG. 9. (Color online) Results for $h(t)$ versus time. 


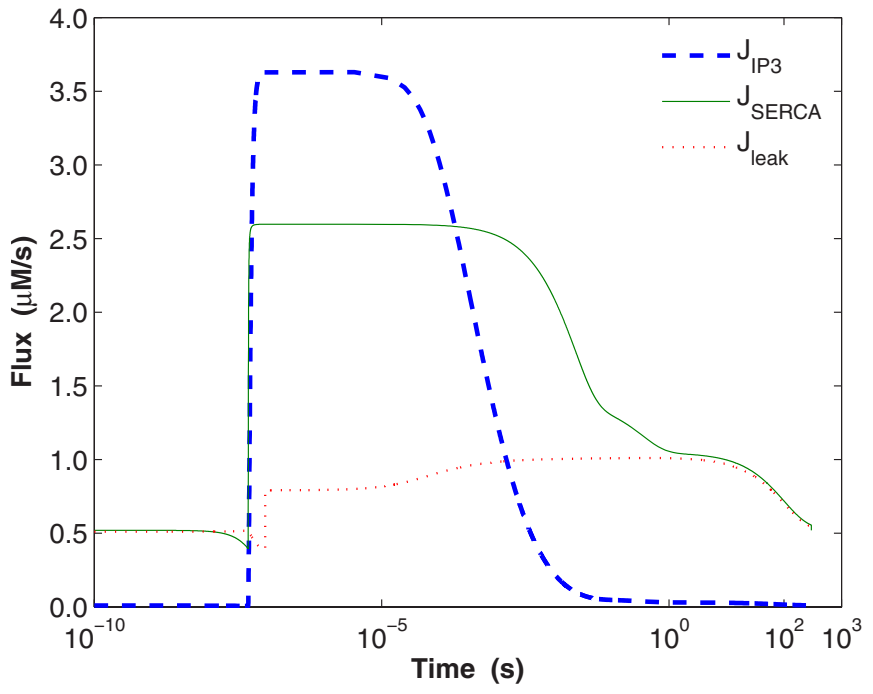

FIG. 10. (Color online) Results for the time dependent flux rates at the ER membrane.

tochondria. The present numerical study constitutes a first step in quantitatively probing the time- and spatially dependent modulation of calcium dynamics through the application of external voltages. A two-step coupled model was used in our analyses. First, the distributed solution for cellular electric fields in response to an externally applied voltage was obtained. This was based on a distributed electrical circuit model coupled with the Smoluchowski equation $[10,22]$ for membrane dynamics. This output was then combined with the continuum Li-Rinzel model [31] for calcium flow. The present approach is quite general and could also usefully be applied to the quantitative analyses of multiple pulsing scenarios and arbitrary external voltage wave forms.

Our results have been shown to be in agreement with the observed calcium dynamics in the absence of extracellular calcium. A modest increase (roughly doubling) of the cellular calcium is predicted that agrees with several reports [8,13-15]. In particular, the applied field of $15 \mathrm{kV} / \mathrm{cm}$ with 60 ns pulse duration makes for a strong comparison. No oscillations are predicted, again in keeping with experimental reports. A quantitative explanation for the lack of such oscil- latory behavior, based on the density dependent calcium fluxes has been provided. A net recovery period of about 5 min following an external $E$ pulse has also been obtained. This agrees with experimental data and sets the limit for a possible memory effect within cells. However, response comparisons for a more diverse parameter set (e.g., other electric fields and pulse durations) need to be carried out for a more complete assessment. This task is currently ongoing. It is possible that at much higher electric fields (e.g., $150 \mathrm{kV} / \mathrm{cm}$ and higher as routinely achieved in our laboratory) the response might become more interesting. The mitochondria and PM would probably get porated, and high transmembrane fields might induce alterations in the IP3 channel functionality. The latter could be analyzed through coupled molecular-dynamics-generalized Born techniques, and efforts in this regard, will be reported elsewhere. Other interesting situations include the application of periodic, repetitive external voltage wave forms with variable frequency and duty cycles for triggering constructive interference with the natural oscillatory cellular calcium phenomena.

Finally, it may also be pointed out that our predictions cannot explicitly be confirmed over the ultrashort time scales due to a lack of experimental time-resolved data. Interesting details of the electric pulse effects, their nonlocality in time, and possible influence on calcium channel response has not been resolved here. Despite the lack of comparison over nanosecond scales, the overall trends and predictions match the available data at longer times. Since the evolution of the dynamical system is sequential, the close match between available experiments and theory is an indirect indicator of the overall validity.

\section{ACKNOWLEDGMENTS}

This work was sponsored in part by an AFOSR-MURI grant (No. F49620-02-1-0320) on Subcellular Responses to Narrowband and Wideband Radio Frequency Radiation managed by Dr. Robert J. Barker. The authors would also like to acknowledge useful and stimulating discussions with J. Wagner (IBM), P. Jung (Ohio University), I. Parker (University of California, Irvine), and P. T. Vernier (University of Southern California).
[1] K. H. Schoenbach, S. J. Beebe, and E. S. Buescher, Bioelectromagnetics (N.Y.) 22, 440 (2001).

[2] R. P. Joshi, Q. Hu, R. Aly, K. H. Schoenbach, and H. P. Hjalmarson, Phys. Rev. E 64, 011913 (2001).

[3] K. H. Schoenbach, F. E. Peterkin, R. W. Alden, and S. J. Beebe, IEEE Trans. Plasma Sci. 25, 284 (1997).

[4] P. T. Vernier, Y. Sun, L. Marcu, C. M. Craft, and M. A. Gundersen, Biophys. J. 86, 4040 (2004).

[5] P. T. Vernier, Y. Sun, L. Marcu, C. M. Craft, and M. A. Gundersen, FEBS Lett. 572, 103 (2004).

[6] Q. Hu, R. P. Joshi, and K. H. Schoenbach, Phys. Rev. E 72, 031902 (2005)

[7] Q. Hu, S. Viswanadham, R. P. Joshi, K. H. Schoenbach, S. J.
Beebe, and P. F. Blackmore, Phys. Rev. E 71, 031914 (2005).

[8] S. J. Beebe, P. M. Fox, L. J. Rec, L. K. Willis, and K. H. Schoenbach, FASEB J. 17, 1493 (2003).

[9] S. J. Beebe, P. M. Fox, L. J. Rec, K. Somers, R. H. Stark, and K. H. Schoenbach, IEEE Trans. Plasma Sci. 30, 286 (2002).

[10] R. P. Joshi, Q. Hu, K. H. Schoenbach, and S. J. Beebe, Phys. Rev. E 69, 051901 (2004).

[11] U. Zimmermann and G. A. Neil, Electromanipulation of Cells (CRC, Boca Raton, FL, 1996).

[12] J. Deng, K. H. Schoenbach, E. S. Buescher, P. S. Hair, P. M. Fox, and S. J. Beebe, Biophys. J. 84, 2709 (2003).

[13] P. T. Vernier, Y. Sun, L. Marcu, S. Salemi, C. M. Craft, and M. A. Gundersen, Biochem. Biophys. Res. Commun. 310, 286 
(2003).

[14] S. J. Beebe, P. F. Blackmore, J. White, R. P. Joshi, and K. H. Schoenbach, Physiol. Meas 25, 1077 (2004).

[15] J. A. White, P. F. Blackmore, K. H. Schoenbach, and S. J. Beebe, J. Biol. Chem. 279, 22964 (2004).

[16] P. Bernardi and V. Petronilli, J. Bioenerg. Biomembr. 28, 131 (1996).

[17] F. Ichas and J. P. Mazat, Biochim. Biophys. Acta 1366, 33 (1998).

[18] K. H. Schoenbach, R. P. Joshi, J. Kolb, N. Chen, M. Stacey, P. Blackmore, E. S. Buescher, and S. J. Beebe, Proc. IEEE 92, 1122 (2004).

[19] H. P. Schwan, Adv. Biol. Med. Phys. 5, 147 (1957).

[20] M. Bootman, M. Berridge, and P. Lipp, Cell 91, 367 (1997); M. J. Berridge, M. D. Bootman, and H. L. Roderick, Nat. Rev. Mol. Cell Biol. 4, 517 (2003).

[21] M. Berridge, M. D. Bootman, and P. Lipp, Nature (London) 395, 645 (1998).

[22] R. P. Joshi, Q. Hu, and K. H. Schoenbach, IEEE Trans. Plasma Sci. 32, 1677 (2004).

[23] C. Taylor, Biochim. Biophys. Acta 1436, 19 (1998).

[24] S. Patel, S. Joseph, and A. Thomas, Cell Calcium 25, 247 (1999).

[25] D. Mak, S. McBride, V. Raghuram, Y. Yue, S. Joseph, and J. Fosken, J. Gen. Physiol. 115, 241 (2000).

[26] X. P. Sun, N. Callamaras, J. Merchant, and I. Parker, J. Physiol. (London) 509, 67 (1998).

[27] J. S. Marchant and I. Parker, EMBO J. 20, 65 (2001).

[28] J. Sneyd and M. Falcke, Prog. Biophys. Mol. Biol. 89, 207 (2005).

[29] A. Bugrim, R. Fontanilla, B. B. Eutenier, J. Keizer, and R. Nuccitelli, Biophys. J. 84, 1580 (2003).

[30] G. W. De Young, and J. Keizer, Proc. Natl. Acad. Sci. U.S.A. 8920, 9895 (1992).

[31] Y. X. Li and J. Rinzel, J. Theor. Biol. 166, 461 (1994).

[32] M. S. Jafri and J. Keizer, Biophys. J. 69, 2139 (1995).

[33] Y. Tang, J. L. Stephenson, and H. G. Othmer, Biophys. J. 70, 246 (1996).

[34] A. Atri, J. Amundson, D. Clapham, and J. Sneyd, Biophys. J. 65, 1727 (1993).

[35] M. B. Falcke, J. D. Lechleiter, and J. L. Hudson, Physica D
129, 236 (1999).

[36] J. Sneyd and L. V. Kalachev, Cell Calcium 15, 289 (1994).

[37] A. E. Bugrim, A. M. Zhabotinsky, and I. R. Epstein, Biophys. J. 73, 2897 (1997).

[38] M. Falcke, L. S. Tsimring, and H. Levine, Phys. Rev. E 62, 2636 (2000).

[39] M. Falcke, Biophys. J. 84, 42 (2003).

[40] S. Swillens, P. Champeil, L. Combettes, and G. Dupont, J. Physiol. (London) 509, 67 (1998).

[41] J. W. Shuai and P. Jung, Phys. Rev. Lett. 88, 068102 (2002).

[42] J. W. Shuai and P. Jung, Proc. Natl. Acad. Sci. U.S.A. 100, 506 (2003).

[43] J. Keizer and G. D. Smith, Biophys. Chem. 72, 87 (1998).

[44] J. Keizer, G. D. Smith, S. Ponce-Dawson, and J. E. Pearson, Biophys. J. 75, 595 (1998).

[45] L. L. Haak, L. Song, T. F. Molinski, I. N. Pessah, H. Cheng, and J. T. Russell, J. Neurosci. 21, 3860 (2001).

[46] R. W. Zwanzig, Lectures in Theoretical Physics (Interscience, New York, 1961).

[47] V. Sridhara, R. P. Joshi, and K. H. Schoenbach, Biochem. Biophys. Res. Commun. 348, 643 (2006).

[48] J. Wagner, C. P. Fall, F. Hong, C. E. Sims, N. L. Allbritton, R. A. Fontanilla, I. I. Moraru, L. M. Loew, and R. Nuccitelli, Cell Calcium 35, 433 (2004).

[49] Z. Vasilkoski, A. T. Esser, T. R. Gowrishankar, and J. C. Weaver, Phys. Rev. E 74, 021904 (2006).

[50] Y. Sun, P. T. Vernier, M. Behrend, J. Wang, M. M. Thu, M. A. Gundersen, and L. Marcu, J. Biomed. Opt. 11, 024010 (2006).

[51] D. A. Cherepanov, B. A. Feniouk, W. Junge, and A. Y. Mulkidjanian, Biophys. J. 85, 1307 (2003).

[52] V. A. Parsegian, Nature (London) 221, 844 (1969).

[53] M. Tarek, Biophys. J. 88, 4045 (2005).

[54] J. Wagner, Y. X. Li, J. Pearson, and J. Keizer, Biophys. J. 75, 2088 (1998).

[55] J. W. Putney, Capacitive Calcium Entry (Landes Biomedical, Austin, TX, 1997).

[56] J. W. Putney, J. Cell Biol. 169, 381 (2005).

[57] G. J. Barritt, Biochem. J. 337, 153 (1999).

[58] E. S. Buescher, R. R. Smith, and K. H. Schoenbach, IEEE Trans. Plasma Sci. 32, 1563 (2004). 